\title{
Energy Efficient Fuzzy Cost-Effective Routing for Transmission of Critical Physiological Parameters in Wireless Body Area Network under Emergency Scenarios
}

\author{
V. Navya, P. Deepalakshmi
}

\begin{abstract}
Wireless body area networks with routing and collaborative fuzzy mechanisms for network analysis have become more efficient in today's healthcare technology. In this article, a novelthreshold-based probability theory and fuzzy logic cost-effective routing technique is proposed that depends on location and residual energy attributes to reduce the overall energy consumption among the sensing nodes and increasing network lifetime. Attributes such as energy and distance are considered for generating if-then rules and membership functions. A fuzzy conditional reasoning is performed using interference mechanism and a defuzzification methodology is applied on the computed cost value to make an efficient choice. Mamdani-Fuzzy logic toolbox in matrix laboratory is used to evaluate the simulation performance of the proposed method with that of other existing conventional methods. From the results obtained, it is observed that for the different metrics, the proposed technique provides improvements in terms of energy efficiency, stability period and network lifetime.
\end{abstract}

Keywords: wireless body area networks; WBAN, fuzzy logic cost-effective technique, threshold-based probability theory, fuzzy conditional reasoning; distance, energy efficiency, healthcare technology.

\section{INTRODUCTION}

Towards the rapid growth and development in Wireless Body Area Networks (WBAN) innovation, today's people need a sound healthcare to improve their life style. Technologies in WBAN get tremendous attention in e-healthcare, ubiquitous and pervasive healthcare monitoring, emotion detection and so on to monitor and diagnose the patient health condition. WBAN consists of different types of implantable and wearable bio-sensors like Electro-Cardio-Gram(ECG), Electro-Encephalo-Gram (EEG), pulse oximeter, motion sensors, glucose sensors etc. that are worn on the patient's body and communicate patients real time health data with each other wirelessly and reliably to the nearby neighboring nodes or Base station (BS) as illustrated in Fig. 1.

In WBAN communication, there exist numerous technical problems such as battery recharge, distance coverage, height of transceiver antenna, heterogeneous data transfer rates etc. One of the critical constrained and challenging tasks is battery powers utilization in the

Revised Manuscript Received on December 16, 2019

* Correspondence Author

Dr. V. Navya*, Dept. of ECE, East Point College of Engineering and Technology, Vigro Nagar Post, Bengaluru, India.mail2navyas@ gmail.com

Dr. P. Deepalakshmi, Dept. of CSE, Kalasalingam Academy of Research and Education, Virudhunagar, India. Deepa.kumar@klu.ac.in implanted devices. Bio-sensor that is placed into the human body is difficult to recharge or replace it. Another factor that effects is network or device lifetime in WBANs. Transmission of real time-varying information and significant communication range between communicating and neighboring nodes cause high energy consumption in the network. Threshold-based energy efficient routing solutions with fuzzy mechanisms are of great significance to reduce network and sensor nodes energy consumption and thereby extend the total system lifetime. But transmission with high Quality of Service (QOS) parameters such as less interference, high packet delivery ratio (PDR) and throughput, less energy consumption, less delay, high data and path reliability etc. are the ultimate requirements depending on their application fields. Normal ranges of various bio-medical sensors are Blood Pressure (BP)-12/80 mmhg, Body Temperature (BT) $-36.5^{\circ} \mathrm{C}-37^{0} \mathrm{C}$, Heart Rate (HR)-60-100 bits/min, Respiration Rate (RR)- at rest 12-16 breaths/min, EEG-gamma waves frequency -30 to

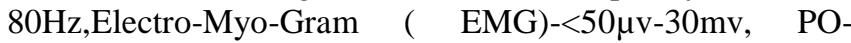
90-100\%,Blood Sugar Level (BSL)- before meal and after meal-4-7mmol/1,5-9 mmol/1 , are adopted from different literature [1] as well as after medical consultation. Normal range may be different for some people based on age/gender but it is acceptable by majority of population.

In this paper, a novel threshold-based data transmission mechanism that uses threshold and fuzzy concept for routing is proposed. The proposed mechanism calculates the cost value for its neighboring nodes based on the amount of energy needed for transmission and distance between the communicating nodes. Fuzzy theory cost value-based routing concept maintains records on cost value of each node and predict the nodes future behavior to improve network lifetime. To evaluate the network performance of the proposed work based on the threshold data and the corresponding cost effective routing strategy, various simulation results are conducted.

In this paper, we propose a novel fuzzy based energy efficient routing technique to healthcare results to the patients. Further, this paper is arranged in the following way: Section 2 gives explanation to related works, Section 3 describes materials and method, Section 3 deals with fuzzy-based cost value, Section 5 deliberates on simulation results and provides a summary in the end, and Section 6 concludes this research work with future enhancement.

Published By: 


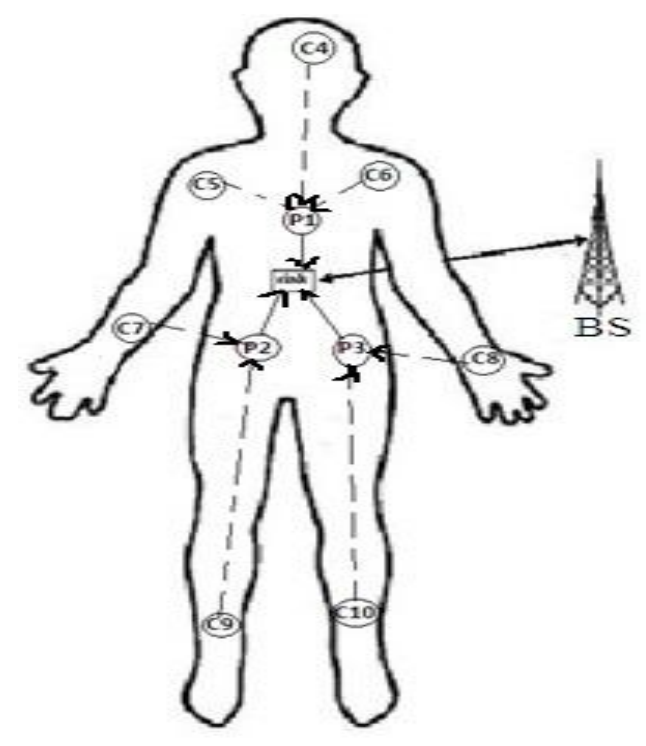

Fig. 1 Location of bio-sensors on human body

\section{RELATED WORK}

Existing routing protocols in literature [2-4] used multi-objective parameters to select the efficient neighboring node to carry out the data transmission depending on minimum and maximum value of metric. For resolving routing and energy issues, different methodologies have been employed to minimize the single node energy or the average energy consumed of all the nodes, or load distribution among the nodes available in the network. Some of the routing protocols with conventional and fuzzy-based approaches are discussed below.

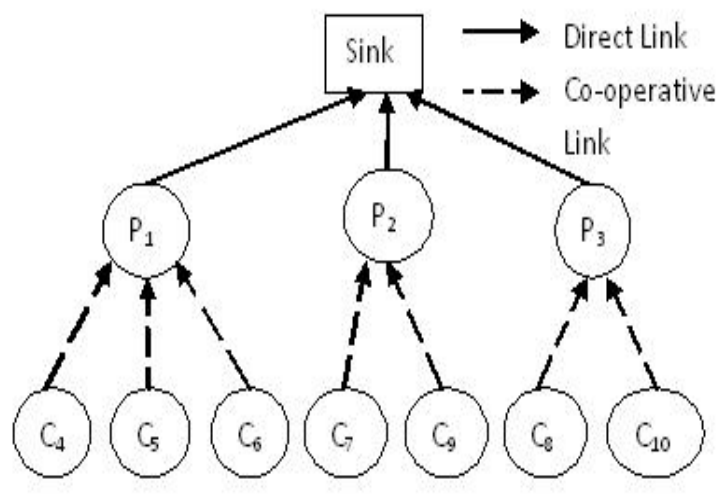

Fig. 2 Corresponding tree topology

The hybrid routing algorithm proposed in [5] used location-based clustering technique to provide efficient covering of sensing area for transmitting data to destination to improve the network lifetime. Cluster head in each region is selected based on fuzzy logic approach that depends on communicating node distance, remaining energy and load distribution parameters. This approach reduced the energy consumed by each sensor node and improved network lifetime and throughput. In the paper [6], a novel trust-based routing that uses probability theory for trust assessment and fuzzy theory for secured routing is proposed. The trust value is calculated probabilistically for every node before transmitting the packet. Using fuzzy theory, node predicts the future behavior to improve performance of overall network. Simulation results showed the improved efficiency in identifying the entrusted nodes and the corresponding attack methodology. In [7], energy efficient threshold based data forwarding technique when the human body is in rest and motion of nodes for heterogeneous WBAN is proposed. Data transmission takes if the threshold condition applied on the generated data and energy level required for effective transmission are satisfied. Tree topology structure of child and parent node is followed and based on cost function metric with high residual energy and less distance, neighboring node is selected for successful data transmission to sink. Comparison result justifies that proposed routing strategy reduces energy consumption among communicating nodes, path loss and delay. This work can also be extended to priority and inter body communication. In the paper [8], authors present a novel distributed pervasive healthcare computing and artificial neural networks for diagnosing internal illnesses and reporting healthcare results to the patients. Patients with internal diseases will have more test results and such reports are time consuming and error prone process for a doctor to diagnose. An Artificial Neural Networks (ANN) classifier at the hospital's server diagnoses 19 different internal illnesses according to the 103 hospital data results from patients and the simplified and understandable results are reported to the patients.

In the reference[9], energy efficient node selection scheme based upon fuzzy logic has been proposed to improve quality of service by considering residual energy, reliability, and latency and utilization factor. Link with the highest link cost is preferred corresponding to different links and made available to the other neighboring nodes until the data reaches the sink. The algorithm has been simulated using Mamdani-fuzzy logic tool and compared with existing protocols in terms of power efficiency and packet delivery ratio. In optimized energy efficient routing algorithm projected in[10], Genetic Algorithm (GA) is used as an optimization technique to the multi-objective cost function with parameters energy, link reliability and path loss for selection of the most efficient route. Inter-BAN communication with multi-hop approach is used for data transmission. Proposed routing technique is compared with existing protocols in terms of energy, packets received and forwarded by intermediate nodes. In [11], the authors propose a novel fuzzy logic cluster formation algorithm, which uses fuzzy concept for the cluster formation. Chance value for each cluster head is found based on energy level and distance between the node parameters. Thus the selected parameters greatly reduce energy consumption in cluster formation and are compared with existing protocol that enhances the network lifetime. In the reference[12], the authors propose a remote patient monitoring algorithm that uses fuzzy concept for the vital parameters being monitored by a patient depending on the condition. Patient to health care personnel ratio is increased by using this approach. As an extension work, environmental condition can also be considered as one of the factor in sensor readings. In [13], an incremental relaying protocol has been proposed that depends on energy consumption and communicating distance parameters. Whenever the first relay fails, the second one is chosen based on cost function metric. Mathematical expressions with different constraints are discussed to improve network lifetime, throughput, and network energy consumption and propagation delay. Results are compared with increase in relay nodes

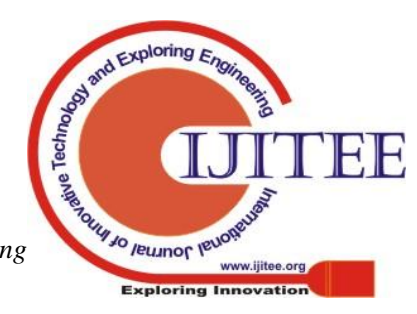


cooperation routing algorithm. Number of hops can be considered as one of the limitation during data transmission. In [14], efficient routing protocol is designed to minimize energy consumption by using cost efficient metric and uses direct path for data transmission of critical data. During data transmission energy consumption is minimized by considering two parameters such as residual and threshold energy for the selection of cluster head to maximize network lifetime. This approach increases the stability period and transfer of more data packets to destination compared to existing techniques. Line of sight parameters can also be considered and evaluated for future work. A thermal-aware routing algorithm in [15], senses the thermal sensors and transmits the data away from these established paths. To minimize energy consumption and increase network lifetime, an efficient routing algorithm is proposed in [16]. The overall network cost and energy level of sensor nodes are addressed and determined by mathematical constraints. For sensor nodes placed nearer to sink, energy is depleted very soon since they were overloaded by data that has to be transmitted.

In our proposed work, we try to reduce energy consumption by using fuzzy logic concept for appropriate node selection and improve throughput by using the threshold approach for the transmission of critical data. Our contribution in this new scheme includes achieving longer stability period and network lifetime to pervasive and ubiquitous healthcare applications which contributes to more number of packets sent and received and also making the sensor nodes to be alive for longer duration of time as the algorithm that we developed here try to minimize energy consumption at sensor and communication level.

\section{MATERIALS AND METHODOLOGY}

\section{A. Proposed framework for threshold and cost value-based routing in WBAN}

Child nodes that are placed away from parent nodes and sink sense the information first and check for threshold conditions as stated in section 1 . If changes are observed after evaluation, the forwarding data is labeled as critical. The child node then checks for its corresponding parent node for further data transmission. A Fuzzy Logic-based Cost value Routing (FLCR) strategy is used to find nearby neighboring node to forward its critical data to destination. This mechanism follows a tree topology concept and provides energy efficiency and high throughput. The proposed frame work as shown in Fig. 3 follows initial, scheduling, evaluation and neighboring nodes selection phases during its routing phase.

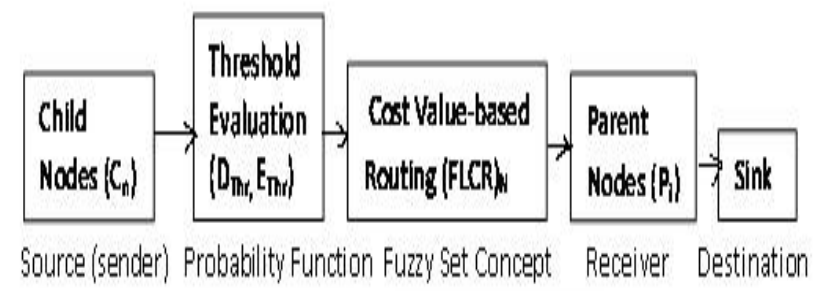

Fig. 3 Architecture for the proposed threshold and cost value-based routing framework

Step 1: Initial phase
Input: $\mathrm{N}=\mathrm{C}_{\mathrm{m}}, \mathrm{P}_{\mathrm{n}}$, Sink locations, $\mathrm{N}$ Distances, threshold levels are set for the data to be forwarded and initial and transmission energy.

For $\mathrm{C}_{\mathrm{m}}=1: 7$

For $\mathrm{P}_{\mathrm{n}}=1: 3$

Calculate the distance (Distance ${ }_{\mathrm{m}, \mathrm{n}-\mathrm{snk}}$ ) between $\mathrm{C}_{\mathrm{m}}$ and $\mathrm{P}_{\mathrm{n}}$ to sink.

End

End

Find all the $\mathrm{C}_{\mathrm{m}}$ and $\mathrm{P}_{\mathrm{n}}$, initial distances from each other to sink, update information to all the node $\mathrm{N}$ in the network

\section{Step 2: Scheduling Phase}

For $\mathrm{m}=1: 7$

For $\mathrm{n}=1: 3$

Check for the sensor data $\left(\right.$ Data $\left._{\text {tranmit }}\right)$ with the fixed threshold value ( Data $a_{\text {threshold }}$ ), changes observed then that node is scheduled and made ready for the transmission.

Evaluate the data thresholds

$$
\text { (Data } \left.\text { tranmit } \sim=\text { Data }_{\text {threshold }}\right) \text {. }
$$

Evaluate the energy thresholds (Energytransmit $>=$ Energythreshold

End

End

\section{Step 3: Evaluation phase}

For $\mathrm{m}=1: 7$

For $n=1: 3$

Calculate the distance (Distance ${ }_{m, n}$ ) with all the neighbors of node $\mathrm{m}, \mathrm{n}$

Calculate the Residual energy (Residual energy $\mathrm{m}_{\mathrm{m}, \mathrm{n}}$ ) with all the neighbors of nodes $m, n$

Calculate the cost value ${ }_{m, n}$ with all the neighbors of nodes $\mathrm{m}, \mathrm{n}$ to sink

End

End

For $\mathrm{m}=1: 7$

For $n=1: 3$

Evaluate the cost value by using Mamdani-Fuzzy logic toolbox in MATLAB.

End

End

Step 4: Node selection phase

If node is not equal to sink node

For $\mathrm{m}=1: 7$

For $n=1: 3$

If distance of node $C_{m}$ to $P_{n}$ (Distance ${ }_{m, n-s i n k}$ ) is less than the specified distance range value and have the lowest cost value, then select that node $\mathrm{N}$ and update it to the nodes list to form the final path go to that node and repeat same steps 
Else

Reject it

End

End

Fig. 4 Pseudo-code for successful transmission of critical data and fuzzy-based routing

Bio-sensors placed on the body uses threshold-based data transmission technique. Each time the sensor node senses the information, comparison of the sensed value $\left(\right.$ Data $\left._{\text {transmit }}\right)$ with the assigned threshold values ( $\left.D a t a_{\text {threshold }}\right)$ is done. These threshold values are the normal values that are mentioned in section I. Data transmission happens only if variations are observed and thereby avoiding unnecessary transmissions. Sink is located at the mid place of the body to collect more critical information from all sensor nodes and transmits to the BS. In case, if sensor node doesn't satisfy the energy condition, Energy $y_{\text {transmit }} \sim=$ Energy $_{\text {threshold }}$ to transmit critical data to sink, data is then transmitted by multihop transmission to sink. If energy condition is satisfied and particular sensor node's energy is decreasing below the threshold value, then single hop/direct transmission takes place. Efficient selection of transmission distance plays a important role in the selection of next hop in case of WBANs. This kind of dynamic routing technique minimizes energy consumption as sensor node selects less distant next hop for further data forwarding by using the Fuzzy Cost Value (FCV) metric in case of finding nearest neighbors to perform multi-hop communication.

Sensor nodes placed on the body have less energy constraints. During the sensor nodes communication, the available battery power should be properly utilized for enhancing network lifetime and connectivity. To preserve energy, we applied tree topology child- parent -sink, based routing scheme using FCV metric that considers distances between the communicating nodes and total amount of energy needed for communication process. Change in the sensor nodes information is updated by the sink in their corresponding routing tables for any further communication based on computed FCV, neighboring nodes decide whether to forward the data further in each round. Identification (ID) of each pair of sensor nodes $\mathrm{m}, \mathrm{n}$ and communicating distances between the sensor nodes $\mathrm{m}, \mathrm{n}$ and sink are given as Distance $_{(m, n-\operatorname{sink})}$ and residual energy (n) refers to the remaining energy of the sensor node that is used for forwarding data. ThenFCV $(\mathrm{P})_{\mathrm{n}}$, for individual neighboring node is computed by the expression as follows:

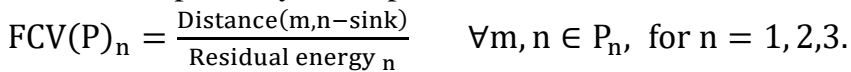

A bio-sensor with least value is chosen to be a communicating node to reach its destination and its identification address is sent to the remaining nodes. Selected sensor node has a maximum remaining energy and is less distant to sink. In the process of communication, the sensor node's aggregated data is transmitted to sink during continuous iterations. Sensor nodes that are very close to sink send their data directly, which results in less energy consumption.

\section{B. Analysis of sensor nodes energy parameters during data transmission}

In this section, we framed a set of energy mathematical statements for direct and multi-hop communications using different energy parameters. Different amount of energy is consumed by sensor nodes placed for intra-body data communication process. Energy spent during the direct communication from child node to parent node is given below.

$\mathrm{E}_{\text {Direct-comm }}=\mathrm{E}_{\mathrm{Tr}-\mathrm{e}}(1)$

where $E_{T r-e}$ is the transmission energy which is given as:

$\mathrm{E}_{\mathrm{Tr}-\mathrm{e}}=\mathrm{P}_{\mathrm{s}} \times\left(\mathrm{e}_{\mathrm{amp}}+\mathrm{E}_{\mathrm{Tx}-\text { elect }}\right) \times$ Distance $_{(\mathrm{m}, \mathrm{n}-\mathrm{sink})^{2}}{ }^{2}(2)$

where, $e_{a m p}$ is the amount of energy utilized in the amplifier circuitry and $E_{T x \text {-elect }}$ is the energy utilized in the electronic components. The size of the packet is expressed as $P_{S}$ and Distance $_{(m, n-\operatorname{sink})}$ is the communicating range connecting sensor nodes and the sink.During multi-hop communication, the energy utilized is specified as follows.

$\mathrm{E}_{\mathrm{mh}}=\mathrm{P}_{\mathrm{s}} \times \mathrm{H}_{\mathrm{n}} \times\left[\mathrm{E}_{\mathrm{Tr}-\mathrm{e}}+\left(\mathrm{E}_{\mathrm{a}}+\mathrm{E}_{\mathrm{Re}-\mathrm{e}}\right) \times \frac{\mathrm{H}_{(\mathrm{n}-1)}}{\mathrm{H}_{\mathrm{n}}}\right]$

In eq. $3, H_{n}$ signifies the hops performed and $E_{a}$ signifies the data aggregation energy. $E_{R e-e}$ signifies the energy utilized in receiving data. Furthermore, it's assumed that $E_{T r-e}=E_{R e-e} \quad$ during initial communication process.

\section{FUZZY-BASED COST VALUE (FCV)}

Probability values cannot be entirely valued to find the appropriate cost value. Consequently, estimation for this metric can be valued from fuzzy interference system approach to make decision for cost value-based routing. The clarification of framing membership functions and creating IF-THEN rules are discussed in the accompanying subsections.

\section{A. Fuzzy Inference System (FIS) for WBAN}

Fuzzy inference maps a given input to an output using logic technique as shown in Fig. 5.Fuzzy Logic-based Cost Routing (FLCR) follows four stages namely: fuzzification, rule assessment, aggregation, and defuzzification. These four stages are followed as a part of FIS to ascertain the possibility of cost values.

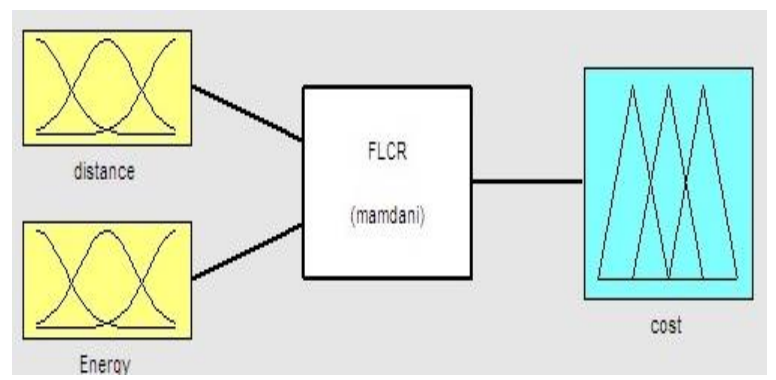

Fig. 5 FLCR system for WBAN with two inputs and one output with 18 rules.

Stage 1: Input of Crisp Value and Fuzzification

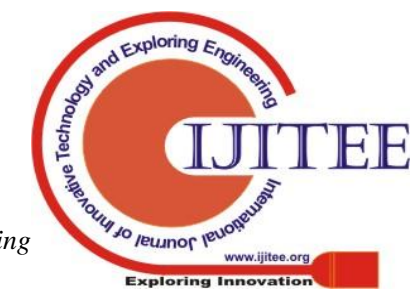


Initially, crisp input information source is forwarded, which signifies two parameters used such as residual energy level and communicating distance between the nodes to FIS. Based on these two crisp input values, value to the membership function is decided, which replicates the convergence position of the estimation of input constants with the degree of the membership function.

\section{Stage 2: Rule Assessment}

After the fuzzification stage has been finished, new fuzzy output set is formed by assigning the membership values to frame IF-THEN principles. Fuzzy IF-THEN principles consists of two input sources and three fuzzy operators (AND, OR, NOT), which essentially opts the minimum/maximum of two membership values, that are utilized to get a solitary number as shown in Fig. 6 .

\section{Stage 3: Aggregation of the Rule Outputs}

Combining together the outputs achieved from employing all the IF-THEN rules (18 rules in our FIS model) defines aggregation process. Each and every rule, that is framed using AND, OR fuzzy logic operators is aggregated. The operators used tend to choose the greatest of our rule assessment values to produce the original aggregate fuzzy set, which is utilized in the next stage.

\section{Stage 4: Defuzzification}

Through de-fuzzification process, true cost value for data transmission is obtained. Mamdani procedures are followed to compute the cost effective significant value. Centroid defuzzification technique is used to locate the approximate cost value for further information communication to sink. In this manner, the Center Of Area (COA) is utilized as a part of the centroid defuzzification, which we can calculate from below equation

$$
\mathrm{Z}_{\mathrm{COA}}=\frac{\sum_{\mathrm{r}=1}^{\mathrm{n}} \mathrm{c}_{\mathrm{r}} \mu\left(\mathrm{c}_{\mathrm{r}}\right)}{\sum_{\mathrm{r}=1}^{\mathrm{n}} \mu\left(\mathrm{c}_{\mathrm{r}}\right)}
$$

where $\mathrm{n}$ represents the list of rules in the fuzzy rule base, $c_{r}$ represents the centroid of the area, $\mu\left(c_{r}\right)$ represents the membership function after the outputs of all rules are combined. By assigning the values we obtained from stage 3 and calculating $C O A$, we decide the cost value for electing the neighboring node for further information transmission to sink. If two Line Of Sight (LOS) nodes have the similar cost value then we select the one near to the sink.

Mamdani-Fuzzy toolbox in MATLAB is used to simulate all the values. Corresponding to all the rules and input variables, their simulated surface values with respect to output cost value are shown in the Fig. 7.

\section{SIMULATION RESULTS AND DISCUSSION}

In order to validate the performance of proposed approach, T-EEFLRPCDT with FCV mechanism is compared with existing conventional routing protocols, M-ATTEMPT and SIMPLE. Simulation results are obtained using MATLAB tool. A two-dimensional area is used for simulation height of $1.4 \mathrm{~m}$ and width of $1 \mathrm{~m}$ corresponding to the human body dimensions. Nodes are located on the front side of the human body to their corresponding $\mathrm{x}$ and $\mathrm{y}$ directions, respectively as indicated in Table I . Initial energy of child nodes $\left(\mathrm{C}_{4}-\mathrm{C}_{10}\right)$ is $0.1 \mathrm{~J}$ each whereas, the parent nodes

with $0.2 \mathrm{~J}\left(\mathrm{P}_{1}-\mathrm{P}_{3}\right)$. The simulation parameters with their values used in the analyses is shown in Table I : .

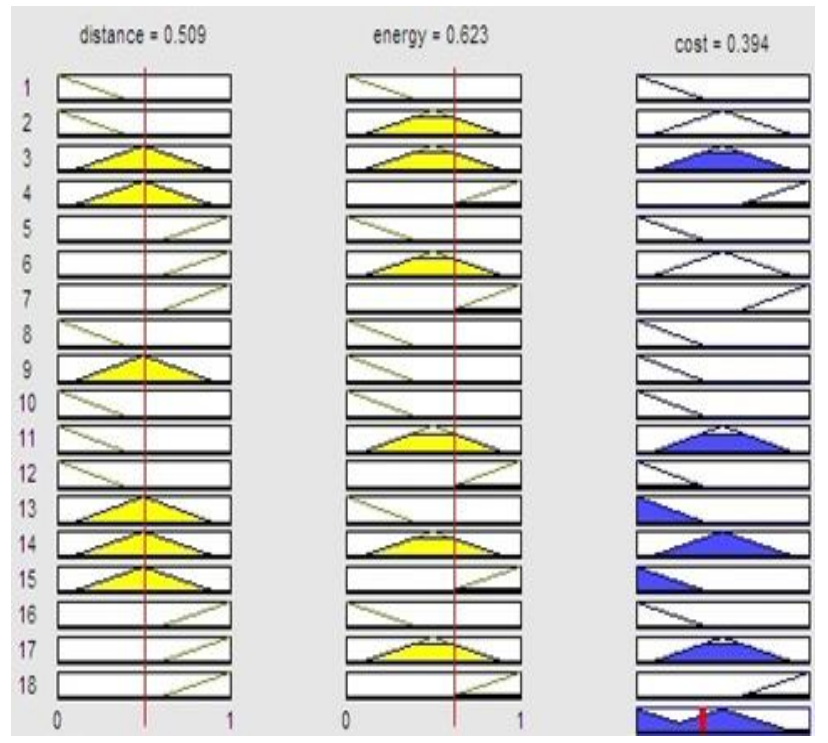

Fig. 6 Fuzzy rules

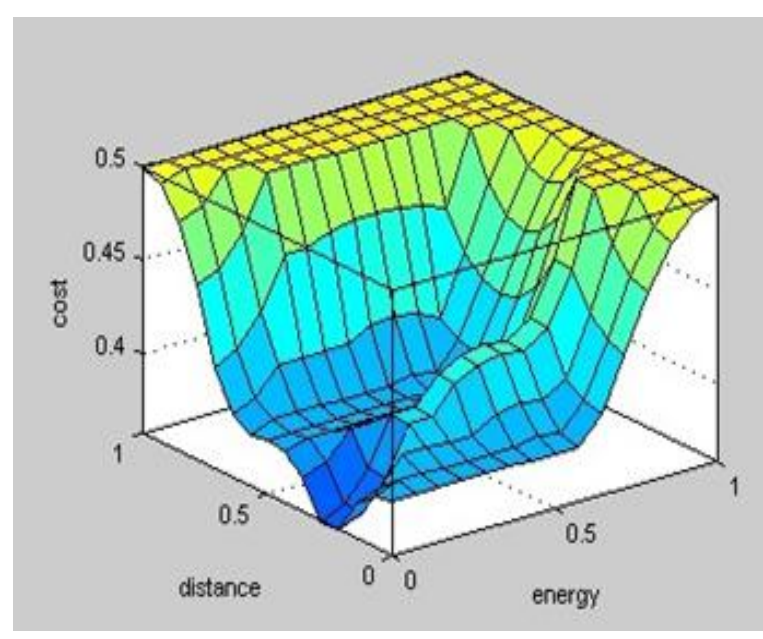

Fig. 7 Surface viewer

Table I : SIMULATION PARAMETERS VALUE

\begin{tabular}{|l|c|c|}
\hline \multicolumn{1}{|c|}{ Parameters } & Value & Units \\
\hline $\mathrm{E}_{\text {Rx-elect }}$ & 36.1 & $\mathrm{~nJ} / \mathrm{bit}$ \\
\hline $\mathrm{E}_{\text {Tx-elect }}$ & 16.7 & $\mathrm{~nJ} / \mathrm{bit}$ \\
\hline $\mathrm{e}_{\mathrm{amp}}$ & 1.97 & $\mathrm{~nJ} / \mathrm{bit} / \mathrm{m}^{\mathrm{n}}$ \\
\hline $\mathrm{E}_{\mathrm{a}}$ & 5 & $\mathrm{~nJ} / \mathrm{bit} / \mathrm{signal}$ \\
\hline $\mathrm{d}_{0}$ & 0.1 & $\mathrm{~m}$ \\
\hline Energy Transmit & 0.2 & $\mathrm{~J}$ \\
\hline Packet size $\left(\mathrm{P}_{\mathrm{s}}\right)$ & 4000 & $\mathrm{bits}$ \\
\hline Frequency $(\mathrm{f})$ & 2.4 & $\mathrm{GHz}$ \\
\hline $\begin{array}{l}\text { Initial } \\
\text { energy }\left(\mathrm{E}_{0}\right)\end{array}$ & 0.5 & $\mathrm{~J}$ \\
\hline
\end{tabular}




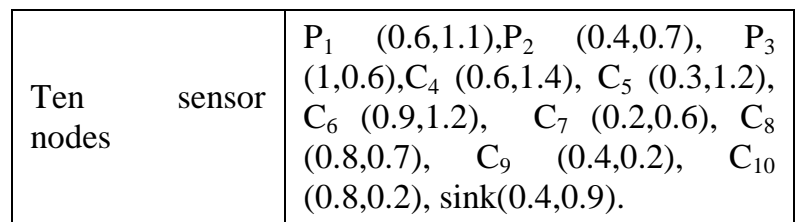

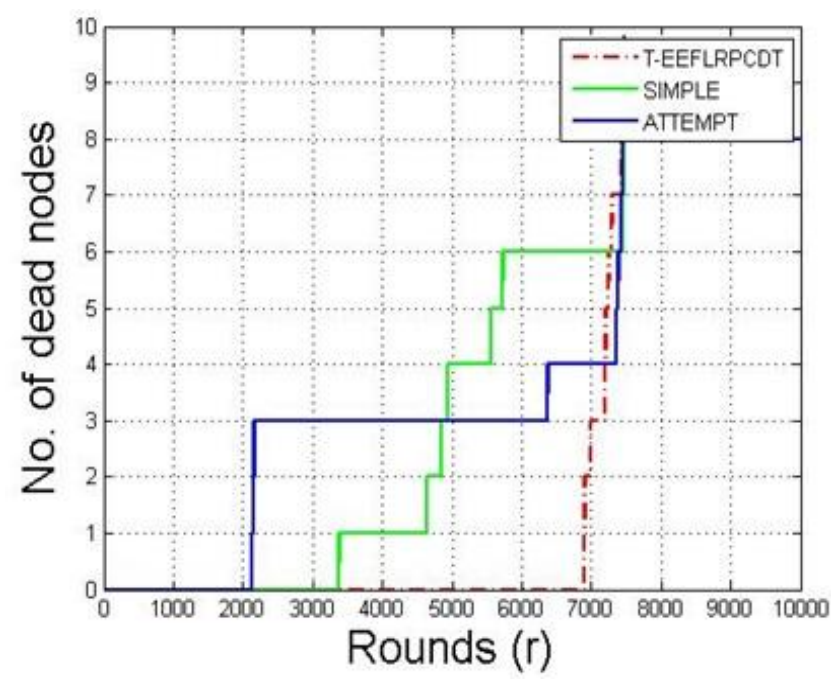

Fig. 8 Analysis of stability period and network lifetime

The start of the iteration to the death of the first node defines the stability period. Similarly, the start of iteration to the death of the last node in the network defines network lifetime. These parameters are important to know the life of the network. From the observation,Fig. 8 shows the stability period and network lifetime of the proposed T-EEFLRPCDT that it is greater than that of existing M-ATTEMPT and SIMPLE. Due to threshold conditions used in data forwarding and thereby avoiding redundant data information, from Fig. 8 we observe that the stability period of T-EEFLRPCDT is attained at $6955^{\text {th }}$ iteration, SIMPLE at $2200^{\text {th }}$ iteration and M-ATTEMPT at $2100^{\text {th }}$ iteration, respectively. Whereas, the network life time remains the same incase of SIMPLE and M-ATTEMPT, respectively.

The number of packets received effectively at destination (sink) defines the throughput of the network. Fig. 9. shows that the number of packets received at sink is more due to FCV based routing mechanism compared to that of SIMPLE and M-ATTEMPT, which follows multihop and forwarder-based approach for data communication respectively. T-EEFLRPCDT shows highest throughput compared to SIMPLE and M-ATTEMPT algorithms, as shown in Fig. 9. More number of alive nodes transmits more number of data packets in the network. The packets sent are not always successfully received due to interferences in line of sight (LOS) communication. From Fig. 9 we observe that the throughput of T-EEFLRPCDT is 14900 packets, SIMPLE having 11200 packets and M-ATTEMPT having 7500 packets, respectively. Huge number of data packets is received due to increased stability period in our proposed T-EEFLRPCDT routing protocol.

Fig. 10. shows M-ATTEMPT, SIMPLE and T-EEFLRPCDT routing protocol's comparison results with respect to remaining energy in the network after each iteration. Strategies such as multi-hop and forwarder-based routing approaches are used in existing protocols that leads to depletion of energy more frequently for the nodes placed very close to the sink. On the other hand, nodes following FCV based routing strategy in tree topology form consumes less energy and stays alive for longer time. Fig. 10 depicts the amount of energy retained in the network as $1 \mathrm{~J}$ and thereafter a gradual decrease in the residual energy in case of T-EEFLRPCDT. Whereas, In SIMPLE and M-ATTEMPT, nodes die early due to heavy traffic and non- uniform load distribution. Thus the residual energy retained is $0.8 \mathrm{~J}$ in the network and thereby a rapid decrease in the energy at the earlier iterations itself.

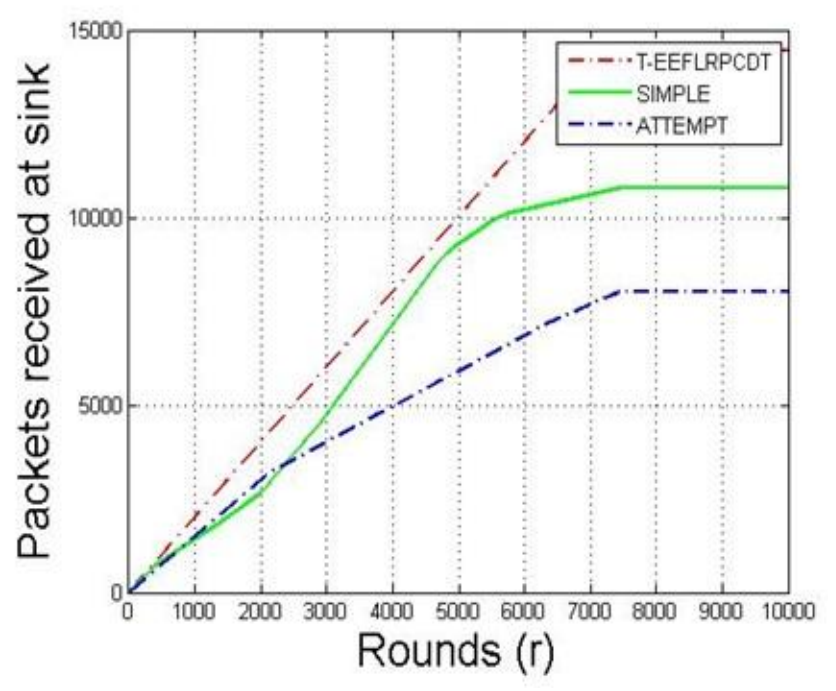

Fig. 9 Analysis of throughput

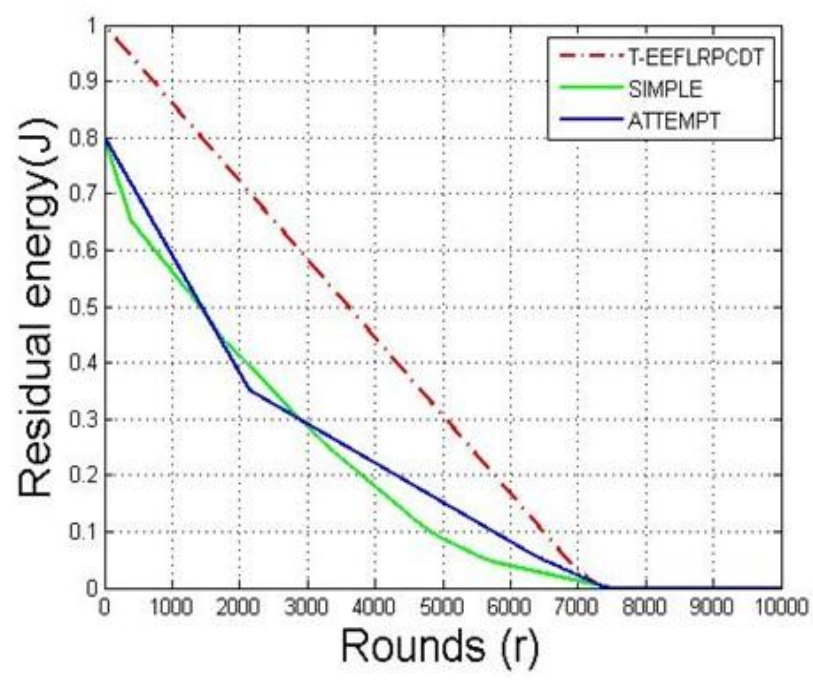

Fig. 10 Analysis of Residual energy

The graphs shown in Fig. 6 - 10) illustrate the results generated using Mamdani FIS being interfaced with the routing concept. Since the decision whether the transmission should be scheduled or not, is made based on the important network quality parameters, the chance of the transmissions colliding with each other reduces. Fuzzy logic has been used in our work as it is very flexible and is based on linguistic variable input and output. In addition to the fuzzy logic, we used threshold-based model to model the input and output data to obtain improvised decision. The graphs indicate the decision made based on the input parameters.

Published By:

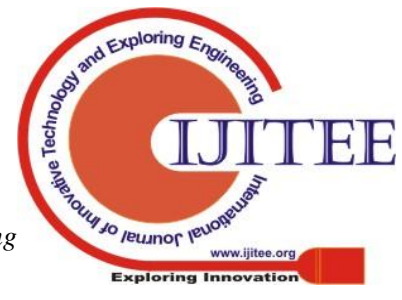




\section{CONCLUSION}

In our work, we addressed energy which is one of the major issues of WBANs. We approached to resolve the issue by considering a threshold based energy efficient routing scheme T-EEFLRPCDT for the sensor nodes placed on human body forming WBANs to overcome associated energy loss during data transmission process. Our work concentrates on providing a scheme to mitigate intra-WBAN communication. The proposed scheme uses threshold and fuzzy logic to deduce a decision for data transmission process. Important network parameters like residual energy and distance between communicating nodes have been considered as the major ones to come up with the decision that reflects the cost value of a communication node. The fuzzy logic results showed that data transmission decision based on the cost value will help us to reduce the energy wastage with other co-existing sensor nodes. Fuzzy logic has proven to be a great way to deal with uncertainty and partial truth input values.

In this work, we considered Mamdani inference system to keep the process simple. The method can also be implemented by using Sugeno inference method. For the membership functions, we considered triangular membership function to assign the degree of membership to the input parameters. Functions such as bell shaped, Gaussian, trapezoidal, etc can be used as membership functions. The proposed scheduling scheme is only for intra-WBAN interference. In inter-WBAN, the interference is due to more than one WBAN existing in close proximity of each other. This approach can be extended to inter-WBAN communication also.

\section{REFERENCES}

[1]. Chintapalli, S. (2015). Transmission Scheduling Using Adaptive Neuro-Fuzzy Inference System For Minimizing Interference in Wireless Body Area Networks (WBANs) (Doctoral dissertation, University of Cincinnati).

[2]. Kim, B. S., Kim, K. H., \& Kim, K. I. (2017). A Survey on Mobility Support in Wireless Body Area Networks. Sensors, 17(4), 797.

[3]. Kumar, A., \& Raj, M. V. (2017). A Comprehensive Survey of QoS-Aware Routing Protocols in Wireless Body Area Networks. International Journal of Advanced Research in Computer and Communication Engineering, 6(1), 166-173.

[4]. Movassaghi, S., Abolhasan, M., Lipman, J., Smith, D., \&Jamalipour, A. (2014). Wireless body area networks: A survey. IEEE Communications Surveys \& Tutorials, 16(3), 1658-1686.

[5]. Maurya, S., \& Daniel, A. K. (2014, December). An energy efficient routing protocol under distance, energy and load parameter for heterogeneous wireless sensor networks. In Information Technology (ICIT), 2014 International Conference on (pp. 161-166). IEEE.

[6]. Pugalendhi, G., Velusamy, D., Paul, A., \& Kim, K. J. (2017) Fuzzy-based trusted routing to mitigate packet dropping attack between data aggregation points in smart grid communication network. Computing, 99(1), 81-106.

[7]. Navya, V., \& Deepalakshmi, P. (2017, March). Mobility supported threshold based stability increased throughput to sink using multihop routing protocol for link efficiency in wireless body area networks (M-TSIMPLE). In Intelligent Techniques in Control, Optimization and Signal Processing (INCOS), 2017 IEEE International Conference (pp. 1-7). IEEE.

[8]. Bayraktar, C., Karan, O., \&Gümüşkaya, H. (2011). Diagnosing internal illnesses using pervasive healthcare computing and neural networks. Procedia Computer Science, 3, 584-588.

[9]. Saini, M., \&Pandove, G. (2017). A Research Article on Efficient Next-Node Selection Algorithm for WBAN by Using Fuzzy Logic. International Journal of Computational Intelligence Research, 13(8), 1977-1990.

[10]. Kaur, N., \& Singh, S. (2017). Optimized cost effective and energy efficient routing protocol for wireless body area networks. Ad Hoc Networks, 61, 65-84.
[11]. Mhemed, R., Aslam, N., Phillips, W., \& Comeau, F. (2012). An energy efficient fuzzy logic cluster formation protocol in wireless sensor networks. Procedia Computer Science, 10, 255-262.

[12]. Billones, R. K. C., Vicmudo, M. P., \&Dadios, E. P. (2015). Fuzzy inference system for remote health monitoring using wireless body area networks. In International Conference on Humanoid, Nanotechnology, Information Technology, Communication and Control, Environment and Management(HNICEM) 2015 by IEEE, DOI:10.1109/HNICEM. 015.7393231, 9-12 Dec.

[13]. Liao, Y., Leeson, M. S., Higgins, M. D., \& Bai, C. (2016, October). An incremental relay based cooperative routing protocol for wireless in-body sensor networks. In Wireless and Mobile Computing, Networking and Communications (WiMob), 2016 IEEE 12th International Conference on (pp. 1-6). IEEE.

[14]. Sethi, D., \& Bhattacharya, P. P. (2016, February). A Study on Energy Efficient and Reliable Data Transfer (EERDT) Protocol for WBAN In Computational Intelligence \& Communication Technology (CICT), 2016 Second International Conference on (pp. 254-258). IEEE.

[15]. Nadeem, Q., Javaid, N., Mohammad, S. N., Khan, M. Y., Sarfraz, S., \& Gull, M. (2013, October). Simple: Stable increased-throughput multi-hop protocol for link efficiency in wireless body area networks. In Broadband and Wireless Computing, Communication and Applications (BWCCA), 2013 Eighth International Conference on (pp. 221-226). IEEE.

[16]. Javaid, N., Abbas, Z., Fareed, M. S., Khan, Z. A., \&Alrajeh, N. (2013). M-ATTEMPT: A new energy-efficient routing protocol for wireless body area sensor networks. Procedia Computer Science, 19, 224-231.

[17]. Yousaf, S., Ahmed, S., Akbar, M., Javaid, N., Khan, Z. A., \&Qasim, U. (2014, November). Co-CEStat: Cooperative Critical Data Transmission in Emergency in Static Wireless Body Area Network. In Broadband and Wireless Computing, Communication and Applications (BWCCA), 2014 Ninth International Conference on (pp. 127-132). IEEE.

[18]. Yousaf, S., Akbar, M., Javaid, N., Iqba, A., Khan, Z. A., \&Qasim, U. (2014,May). Cemob: Critical data transmission in emergency with mobility support in wbans. In Advanced Information Networking and Applications (AINA), 2014 IEEE 28th International Conference on (pp. 915-919). IEEE.

[19]. Sandhu, M. M., Javaid, N., Jamil, M., Khan, Z. A., Imran, M., Ilahi, M., \& Khan, M. A. (2015). Modeling mobility and psychological stress based human postural changes in wireless body area networks. Computers in Human Behavior, 51, 1042-1053.

[20]. Javaid, N., Ahmad, A., Nadeem, Q., Imran, M., \& Haider, N. (2015). iM-SIMPLE: iMproved stable increased-throughput multi-hop link efficient routing protocol for Wireless Body Area Networks. Computers in Human Behavior, 51, 1003-1011.

\section{AUTHORS PROFILE}

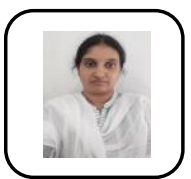

Dr. V. Navya has received $\mathrm{PhD}$ in Electronics and Communication Engineering from Kalasalingam Academy of Research and Education, India. Now she is an Associate Professor in the Department of Electronics and Communication Engineering, East Point College of Engineering and Technology. She has 5 years of teaching experience in Academics and Research. Her area of interests includes wireless body area networks, wireless communication, soft computing and pervasive computing techniques. Owned a membership in IEEE and ISTE societies. Acted as a paper reviewer for International conferences.

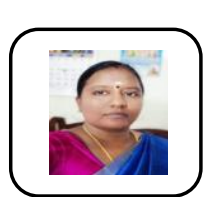

Dr.P.Deepalakshmi is currently working as a Professor in Department of Computer Science and Engineering at Kalasalingam Academy of Research and Education (KARE), Virudhunagar, Tamil Nadu, India. She is also serving as Dean, School of Computing. Her research interest includes Optimization Techniques, Network Routing, Distributed Computing, Network Security, Data Analytics, Machine Learning Techniques. She also takes care of KARE ACM student chapter as faculty mentor. Contact her at deepa.kumar@klu.ac.in 